\title{
ITERATES OF HOLOMORPHIC SELF-MAPS OF THE UNIT BALL IN HILBERT SPACE
}

\author{
ADAM STACHURA
}

\begin{abstract}
An example is given of a biholomorphic self-mapping $T$ of the unit ball in infinite-dimensional Hilbert space satisfying $0=\lim \inf _{n}\left\|T^{n}(0)\right\|$ $<\lim \sup _{n}\left\|T^{n}(0)\right\|=1$.
\end{abstract}

1. Introduction. Let $B$ denote an open unit ball in the complex Hilbert space $H$ and let $T$ be a holomorphic self-mapping of the ball $B$ with no fixed points in $B$. The sequence of iterates of such a mapping was considered by several authors. In case $H=\mathbf{C}$ (the complex plane) the basic result comes from the old papers of J. Wolff [15] and A. Denjoy [3] (see also [1]). They showed that the iterates converge to a unimodular constant (uniformly on compact subsets of the unit disc $\Delta)$. Recently it was generalized $[\mathbf{1 1}, \mathbf{1 3}]$ to self-mappings of the unit ball $B \subset \mathbf{C}^{n}$ with $n>1$.

In the infinite-dimensional case analogous results have been obtained in several special cases only $[\mathbf{7}, \mathbf{1 4}]$. It was not quite clear whether or not a similar statement holds for all holomorphic, fixed point free mappings $T: B \rightarrow B$. Our aim in this paper is to construct an example showing that the answer is negative even for automorphisms (i.e. biholomorphic self-mappings of the ball $B$ ).

Recall that $B$ can be furnished with the invariant hyperbolic metric $\rho$ so that every holomorphic mapping $T: B \rightarrow B$ becomes $\rho$-nonexpansive $[\mathbf{5}, \mathbf{1 0}]$. Recently it has been shown that there exist interesting analogies between properties of $\rho$ nonexpansive self-mappings of the ball $B$ and of norm-nonexpansive mappings on the whole space $H[6, \mathbf{6}, \mathbf{1 2}]$. Therefore our question is closely related to a similar problem concerning a norm-nonexpansive, fixed point free mapping $F: H \rightarrow H$. For such a mapping one can show that if $\operatorname{dim} H<\infty$, then $\lim _{n \rightarrow \infty}\left\|F^{n}(x)\right\|=\infty$ for all $x$ in $H$ (see [2 and 16] for even more general results). On the other hand M. Edelstein [4] gave an example of an isometry $F: l^{2} \rightarrow l^{2}$ satisfying the following conditions:

(i) $F$ has no fixed point in $l^{2}$;

(ii) $\left\{F^{n}(0)\right\}$ is norm-unbounded;

(iii) There exists a subsequence $\left\{F^{n_{k}}(0)\right\}$ with $\lim _{k \rightarrow \infty} F^{n_{k}}(0)=0$

(see [4] for details). Here $l^{2}$ denotes the space of all sequences $x=\left(x_{n}\right)$ of complex numbers with the usual Hilbert norm. Let us notice that $F$ can be easily rewritten in the form

(iv) $F(x)=f(x)+a$, where $x \in H, a=F(0)$ and $f$ is a linear isometry onto $l^{2}$.

Received by the editors January 3, 1984.

1980 Mathematics Subject Classification. Primary 47H10, 32H15.

Key words and phrases. Holomorphic mappings, hyperbolic metric, isometric mappings, fixed points. 
Our construction below is based upon this example.

2. Construction of the example. Let the infinite-dimensional complex Hilbert space $H$ be given. Recall first that the unit ball $B \subset H$ is the biholomorphic image of the region (the Siegel upper half-space) $\Omega=\{(\lambda, w) \in \mathbf{C} \times H: \operatorname{Im} \lambda>$ $\left.\|w\|^{2}\right\}$ under the Cayley transform $C$ (see, for instance, [8, Chapter 2, §31]). Notice that $C$ maps the point $(i, 0) \in \Omega$ into the origin. Clearly every holomorphic mapping $T: B \rightarrow B$ can be obtained from a suitable holomorphic mapping $\Phi: \Omega \rightarrow \Omega$ by composition on the right and left by $C^{-1}$ and $C$ respectively.

Now let $K$ be a real Hilbert space such that $H=K \oplus i K$. Denote by $F_{1}$ an isometry of the space $K$ which satisfies properties (i)-(iv) with $l^{2}$ replaced by $K$ (the existence of such an isometry follows immediately from Edelstein's example). Clearly $F_{1}$ can be extended to an isometry $F: H \rightarrow H$ also satisfying (i)-(iv) and, in addition, satisfying

(v) $F(K)=K$.

Now we define the mapping $\Phi: \Omega \rightarrow \Omega$ by

$$
\Phi(\lambda, w)=\left[\lambda+i\|a\|^{2}+2 i\langle f(w), a\rangle, f(w)\right]
$$

$(\langle$,$\rangle denotes the inner product in H)$. It is easy to observe that $\Phi$ is an automorphism of $\Omega$. A little calculation shows that (1) can be rewritten in the form

$$
\Phi(\lambda, w)=\left[\lambda+i\left(\|F(w)\|^{2}-\|w\|^{2}\right)-2 \operatorname{Im}\langle f(w), a\rangle, F(w)\right],
$$

which reduces for $w \in K$ to

$$
\Phi(\lambda, w)=\left[\lambda+i\left(\|F(w)\|^{2}-\|w\|^{2}\right), F(w)\right] .
$$

Hence we obtain

$$
\Phi^{n}(\lambda, w)=\left[\lambda+i\left(\left\|F^{n}(w)\right\|^{2}-\|w\|^{2}\right), F^{n}(w)\right]
$$

for every $w \in K, \operatorname{Im} \lambda>\|w\|^{2}$ and $n=1,2, \ldots$

Set $(\lambda, w)=(i, 0)$ in $(2)$. We have

$$
\Phi^{n}(i, 0)=\left[i+i\left\|F^{n}(0)\right\|^{2}, F^{n}(0)\right] .
$$

From (ii) and (iii) it immediately follows that $\Phi^{n_{k}}(i, 0) \rightarrow(i, 0)$ as $k \rightarrow \infty$ and that $\left\{\Phi^{n}(i, 0)\right\}$ is unbounded in $\Omega$.

Put now $T=C \circ \Phi \circ C^{-1}$. It is easy to observe that $T$ is a fixed point free automorphism of the ball $B$ such that $\limsup _{n}\left\|T^{n}(0)\right\|=1$ and $T^{n_{k}}(0) \rightarrow 0$ as $k \rightarrow \infty$. Thus $T$ is the desired example.

\section{REFERENCES}

1. R. B. Burckel, Iterating analytic self-maps of discs, Amer. Math. Monthly 88 (1981), 396-407.

2. A. Całka, Topological semigroups and a Kirk's question, Colloq. Math. (in press).

3. A. Denjoy, Sur l'iteration des fonctions analytiques, C. R. Acad. Sci. Paris 182 (1926), 255-257.

4. M. Edelstein, On non expansive mappings of Banach spaces, Proc. Cambridge Philos. Soc. 60 (1964), 439447.

5. T. Franzoni and E. Vesentini, Holomorphic maps and invariant distances, North-Holland, Amsterdam, 1980.

6. K. Goebel, Fixed points and invariant domains of holomorphic mappings of the Hilbert ball, Nonlinear Anal. 6 (1982), 1327-1334.

7. K. Goebel and S. Reich, Iterating holomorphic self-mappings of the Hilbert ball, Proc. Japan Acad. Ser. A Math. Sci. 58 (1982), 349-352. 
8. __ Uniform convexity, hyperbolic geometry. and nonexpansive mappings. Marcel Dokker. New York and Basel, 1984.

9. K. Goebel, T. Sekowski and A. Stachura. Iniform convexity of the hyperlolic metric and fixed points of holomorphic mappings in the Hilbert ball. Nonlincar Anal. 4 (1980). 10111021.

10. L. A. Harris, Schwarz Pick systems of pseudometrics for domains in a normed linear space. Advances in Holomorphy, (J. A. Barroso, ed.), North-Holland. Amsterdam. 1979. pp. 345 406.

11. Y. Kubota, Iteration of holomorphic maps of the unit ball into itself. Proc. Amor. Math. Soc. 88 (1983), 476-480.

12. T. Kuczumow and A. Stachura, Extensioms of nonexpansive mappings in the Hilkert bull unth the hyperbolic metric. I, II preprint.

13. B. D. MacCluer, Iterates of holomorphic self maps of the unit ball in $\mathbf{C}^{N}$. Michigan Math. J. 30 (1983), 97-106.

14. T. J. Suffridge, Common fixed points of commuting holomorphic maps of the hyperball. Michigan Math. J. 21 (1974), 309-314.

15. J. Wolff, Sur l'iteration des fonctions, C.R. Acad. Sci. Paris 182 (1926), 42 43. 200201.

16. G. Zhang, Orbits of a non expansive mapping in Banach spaces. Chinese Ann. Math. Sor. A (2) 4 (1983), 171 175 .

InSTytut MATEMATYKI UMCS, PlaC: MARII CURIE-SKLODOWSKIE.J 1. 20-0)31 Liblix. POLAND 